\title{
Writing letters for transgender patients undergoing medical transition
}

Amy Riese, MD

$\mathrm{T}$ ransgender and nonconforming people are estimated to make up $0.3 \%$ to $1.4 \%$ of the population, and these estimates are likely undercounts. ${ }^{1}$ Knowingly or unknowingly, psychiatric and mental health clinicians are caring for transgender patients, and need to become familiar with ways to provide proper clinical care for this often-marginalized population. Being knowledgeable about the requirements for letter writing for patients who are transgender and desire to transition medically is one way that we can assist and affirm these individuals.

The World Professional Association for Transgender Health (WPATH) publishes standards of care (SOC) that discuss the role of mental health professionals during a patient's gender transition. ${ }^{2}$ The initial mental health evaluation should establish if gender dysphoria exists, and not just assume that it does. It is also important to assess whether the patient has had past negative experiences in the treatment setting or a history of trauma, and to evaluate for stressors in social life. Some transgender people may present to mental health professionals solely for the purpose of pursuing gender-related services, and others do not. The transgender person may or may not choose to undergo hormone replacement therapy or surgical transition.

Within the United States, a small percentage of clinicians use the informed consent model and, instead of requiring letters for medical intervention, will conduct an assessment to determine if the patient can provide informed consent about the procedures. But because the WPATH SOC are considered the primary standards and insurance companies will not cover the surgeries without these letters, most surgeons will not accept a patient without this documentation. ${ }^{3}$

\section{Letters for hormone therapy and upper body surgery}

Adults need 1 letter of recommendation from a qualified mental health professional, and the following WPATH criteria must be met: 1) persistent, well-documented gender dysphoria, 2) capacity to make a fully informed decision to consent for treatment, 3) age of majority, and 4) if significant medical or mental health conditions are present, they must be reasonably well-controlled. ${ }^{4}$

The letter should contain identifying characteristics; diagnoses and psychosocial assessment; duration of clinical relationship; type of evaluation or therapy; an explanation that the criteria for hormone therapy have been met/clinical rationale (gender dysphoria, capacity to consent, age of majority, that other mental health conditions are reasonably well-controlled); a statement that informed consent had been obtained; and a statement that the referring clinician is available for coordination of care.

continued

\section{Every issue of CURrent PsychiatRY} has its 'Pearls'

\section{Yours could be found here.}

Read the 'Pearls' guidelines for manuscript submission at MDedge.com/ CurrentPsychiatry/page/pearls. Then, share with your peers a 'Pearl' of wisdom from your practice.
Dr. Riese is Assistant Professor, Department of Psychiatry, University of Toledo College of Medicine and Life Sciences, Toledo, Ohio.

\section{Disclosure}

The author reports no financial relationships with any companies whose products are mentioned in this article, or with manufacturers of competing products.

doi: 10.12788/cp.0159

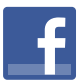

Discuss this article at www.facebook.com/ MDedgePsychiatry 
Understanding

the requirements

of these letters

is one way to

assist and affirm

patients who are

transgender

\section{Letters for lower body surgery}

WPATH recommends letters from 2 mental health clinicians who evaluated the patient. In addition to the criteria set for hormone therapy described above, the SOC recommend 12 months of continuous living in the gender role that is congruent with a patient's gender identity before genital surgery. It is also suggested that the patient undergoes 12 months of hormone therapy before hysterectomy/oophorectomy in transgender men or before orchiectomy in transgender women. ${ }^{4}$

The letter should contain identifying characteristics; diagnoses and psychosocial assessment; duration of clinical relationship; type of evaluation or therapy; criteria for surgery/clinical rationale (gender dysphoria, capacity to consent, age of majority, other health concerns are well-controlled, hormone therapy, real-life experience), informed consent; and availability for coordination of care.

Transgender individuals need clinicians who can provide competent, sensitive health care, and gender affirmation can enhance psychological health.

\section{References}

1. Winter S, Diamond M, Green J, et al. Transgender people: health at the margins of society. Lancet. 2016;388(10042): 390-400.

2. World Professional Association for Transgender Health. Standards of care for the health of transsexual, transgender and gender nonconforming people. 7th version. Published 2012. Accessed July 14, 2021. https://www.wpath.org/ publications/soc

3. Budge SL, Dickey LM. Barriers, challenges, and decisionmaking in the letter writing process for gender transition. Psychiatr Clin N Am. 2016;40(1):65-78.

4. Coleman E, Bocking W, Botzer M, et al. Standards of care for the health of transsexual, transgender and gendernonconforming people. Version 7. International Journal of Transgenderism. 2011;13:165-232. 